\title{
KARL HEINZ GÖLLER (1924-2009) In Memoriam
}

\author{
Janez Stanonik
}

\begin{abstract}
The study is written in memory of Karl Heinz Göller, Professor of English literature at the Regensburg University, who died in Kelheim near Regensburg on 22. April 2009. University of Regenburg was founded in 1967, and Göller was the first Dean of its Faculty of Philosophy. For more than 40 years he worked for the development of good contacts betwen the universities of Regensburg and Ljubljana. Since 2000 he was member of the Advisory Committee of the review Acta Neophilologica. In 1983 he founded the Society of German Mediaevalists, one of the leading societies of German scholars from the whole Germany, and was elected its first president. The study gives report on the development of Göller as a scholar, and his basic achievement in his research, pedagogical work and in university administration.
\end{abstract}

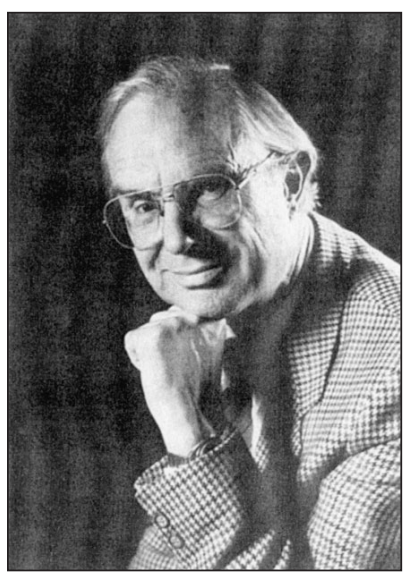

Key words: Karl Heinz Göller, his biography, his research and his pedagogical work; University of Regensburg

Es ist für mich eine traurige Pflicht mit diesem Beitrag das Ableben meines guten Freundes und Berufskollegen Karl Heinz Göller zu melden. ${ }^{1}$ Er starb am 22. April 2009, kurz vor der Vollendung seines 85. Lebensjahres. Die letzten Jahre seines Lebens lebte er zusammen mit seiner Frau Jutta in seinem Haus in Kelheim, einer Stadt auf Ufer der Donau, oberhalb Regensburg. Seit Jahrgang 2000 war Göller Mitglied des Aufsichtsrates unserer Zeitschrift Acta Neophilologica, und das ist Anlass zum Schreiben dieses Nekrologs.

Das Leben Göllers war tief von der Zeit, in welcher er lebte, geprägt. Das 20. Jahrhundert ist durch zwei Weltkriege charakterisiert, die nicht nur Deutschland, sondern auch weite Teile Europas, in Trümmer legte. Die Geschichte das Balkans zeigt für diese

\footnotetext{
${ }^{1}$ Nekrologe zur Erinnerung an Karl Heinz Göller haben geschrieben: Wilhelm BUSSE (Düsseldorf): Karl Heinz Göller (1924-2009), Das Mittelalter XIV (2009), B. 2, S. 162-163. - Richard UTZ (Western Michigan University): In Memoriam Karl Heinz Göller (May 13, 1924 - April 22. 2009), Perspicuitas (Internet-Periodicum für mediaevistische Sprach-, Literatur- u. Kulturwissensschaft, Universität DuisburgEssen) Januar 2010.
} 
Zeit, wie tief die Wurzeln dieser Tragödie lagen. Die Unfähigkeit der Weltpolitker nach dem Ende des Ersten Weltkrieges führte Deutschland in eine tiefe ökonomische Krise, die in dem Wahnsinn des Nationalsozialismus endete. Uns sind noch immer die Erwägungen der deutschen Heeresleitung vor dem Ende des Ersten Weltkrieges unbekannt, die es Lenin ermöglichten nach Russland zu fahren. Wir hoffen, dass in Zukunft die Welt doch neue Auswege für solche Tragödien findet.

\section{1.}

Karl Heinz Göller war geboren am 13. April 1924 in Neheim-Hüsten, einem Orte östlich von Dortmund, im Lande Nordrhein-Westfalen. Seine Ahnan waren Westfalen. Nach der Beendigung der Mittelschulbildung wurde er in der Zeit des Nationalsozialismus in den Deutschen Arbeitsdienst mobilisiert. Während des Zweiten Weltkrieges diente er in der Wehrmacht. Kurz vor dem Ende des Zweiten Weltkrieges geriet er in die englische Kriegsgefangenschaft.

Nach dem Ende des Zweiten Weltkrieges wurde Göller bald aus der englischen Kriegsgefangenschaft freigelassen. Er wollte nun sein Studium an einer Universität verfolgen. Das gelang ihm anfangs jedoch nicht, da die Universitäten mit Studenten überfüllt waren. So überlebte er diese Nachkriegsjahre, die durch Hungersnot und ungenügende Heizung charakterisiert waren, als Volksschullehrer in Limburg an der Lahn (1946-1952). Hier vermählte er sich mit seiner ersten Ehefrau Annerose. Obgleich sie aus einer kommerziellen Familie kam, hatte sie Verständnis für seine intellektuellen Bestrebungen eine Universitätsbildung zu erlangen.

Im Jahre 1953 gelang es endlich Göller sein Studium an der Universität Bonn anzufangen. Er studierte Anglistik unter Professor Walter Franz Schirmer, in seiner Zeit einem der bekanntesten deutschen Anglisten. ${ }^{2}$ Neben Schirmer gab es in Jener Zeit in Bonn noch mehrere wohlbekannte Professoren, die für Göller bedeutend waren, obgleich sie nicht Anglistik unterrichteten: der Literaturhistoriker Günther Müller mit Vorlesungen über die deutsche Literatur des Mittelalters, die Philosophen Theodor List und Erich Rothacker, und der Romanist Ernst Robert Curtius, der sich auch für die Poesie T. S. Eliots interessierte. Sehr debeutend für Göllers wissenschaftliche Entwicklung war auch der Altgermanist Werner Richter. Unter Göllers Mitschülern finden wir viele Namen, die später in Deutschland bekannt waren: Arno Esch, Ulrich Broich, Theo Golpers, Theo Stemmler, Rudolf Sühnel und Erwin Wolff.

Schon nach dem ersten Jahre seines Universitätsstudiums erweckte Göller die Aufmerksamkeit seines Universitätslehrers, Professor Schirmers: Göller war an der englischen Poesie des XVIII. Jahrhunderts interessiert. Das war auch das Gebiet in welchem Schirmer zu dieser Zeit arbeitete. In 1955 verteidigte Göller bei Schirmer mit Erfolg seine Doktorarbeit Die Entwicklung von Thomsons Weltbild. Der englische Dichter James Thomson hat in den Jahren 1726-1730 mit seinem Gedicht The Seasons

\footnotetext{
${ }^{2}$ Walter Franz Schirmer, geboren in Düsseldorf am 18. Dezember 1888, war 1925-1929 Professor der Anglistik in Bonn, 1929-1932 Professor in Tübingen, und nach 1932 in Berlin. Seit 1946 war er wieder in Bonn, wo er 1957 emeritierte, doch blieb er wissenschaftlich tätig bis zum Ende seines Lebens. In den Jahren 1942-1964 war er Mitherausgeber der Zeitschrift Anglia. Er starb in Bonn am 22, März 1984.
} 
das Gefühl für die Schönheit des Lebens auf dem englischen Lande erweckt und so die Bewegung der englischen Vorromantik ins Leben gebracht. Ein Jahr nach dieser Dissertation, in 1956, wurde Göller zum wissensshaftlichen Assistenten an der Universität Bonn ernannt. Ein Jahr darauf, 1957, wurde Professor Schirmer emeritiert, doch blieb er noch weiter wissensshaftlich tätig, diesmal in der englischen Literatur der Mittelalters. 1963 erwarb sich Göller mit der Habilitationsarbeit König Arthur in der englischen Literatur des späten Mittelalters den Titel des habilitierten Anglisten.

Im Jahre 1963 erhielt Göller den Posten eines Universitätslehrers für Anglistik in Göttingen. Doch blieb er in Göttingen nur vier Jahre, da er sich bald nach seiner Ankunft in Göttingen entschloss nach Regensburg weiterzufahren. Göttingen war für Göller nur ein kurzes Intermezzo in seiner Universitätslaufbahn.

2.

Die Universität Regensburg wurde 1962 gegründet. Das war eine der ersten Universitäten, die in Deutschland nach dem Zweiten Weltkrieg kreiert wurden. Die Wahl des Ortes war sehr glücklich: sie verband die neue Hochschulanstalt mit einer der ältesten Städte Deutschlands. Der Lehrbetrieb an der neuen Universität begann Anfang November 1967.

Am 7. Oktober 1966 erhielt Göller in Göttingen die Verfügung dass er mit 1. Mai 1967 als Lehrstuhlinhaber für Anglistik an der Universität Regensburg ernannt war, das ist: ein Semester vor der Eröffnung der neuen Universität. Er kam Anfang Mai nach Regensburg als einer der ersten Professoren an der neuen Universität. Schon Anfang Juni wurde er, für die Dauer von einem Jahr, zum Dekan der neugegründeten Philosophischen Fakultät ernannt. Göller kann deshalb mit Recht der Gründungsdekan der Philosophischen Fakultät Regensburg genannt werden. Verbunden mit dieser Ehre waren jedoch schwere Pflichten, die den Bau des neuen Universitätsgebäudes und die Gründung der Universitätsbibliothek betrafen. Daneben gab es zahllose Konferenzen an der Fakultät, beim Rektorat der Universität Regensburg und in den Ämtern des Landes Bayern und des Staates. So geschah Göller was vielen von seinen Zeitgenossen zuteil wurde: man überforderte ihn mit Pflichten, die kaum noch zu tragen waren. Der Staat riskierte auf diese Weise ganze Generationen von seinen Zeitgenossen um das Land möglichst bald aus der Nachkriegsnot zu retten.

Die Gründung der neuen Universität war verbunden mit der Lösung der Frage, wie sich die neue Universität den Erfordernissen ihrer Zeit anpasen sollte. Göller war in seinen Ansichten vorsichtig konservativ. Er sah ein, dass das alte System der Ordinarier, die an den Universitäten ihre wissensshaftliche Arbeit pflegten, dabei aber den Unterricht als eine nebensächliche Pflicht betrachteten, für die Universitäten nach dem Zweiten Weltkrieg nicht länger annehmbabr war. Er betrachtete die Universität vor allem als die höchste Unterrichtsanstalt, die jedoch ihre alten Verdienste für die Wissenschaft nicht verlieren sollte. Gleichzeitig mit dieser Stellungnahme verteidigte er den Unterricht der englischen Sprache und Literatur an den deutschen Universitäten, wo die Anglistik eine der Zeit gemässe Stelle haben sollte. Er forderte auch, dass in Deutschland die Mittelalterforschung ihre alte Bedeutung behalten sollte. Die Interessen 
der Mittelalterforschung unterstützte er auch mit seinen Bemühungen für die Gründung des Mediävistenverbandes, die 1983 mit der Gründung des Verbandes mit Erfolg gekrönt waren. Als Anerkennung für seine Bemühungen wurde Göller zum ersten Presidenten des neuen Vereins gewählt und behielt diesen Ehrenposten noch mehrere Jahre, worauf er zum Vizepresidenten des Vereines gewählt wurde. Heute verbindet der Verein die Mediävistenforscher aus ganz Deutschland und hat ein eigenes wissenschaftliches Organ, die Zeitschrift Das Mittelalter. Diese Zeitschrift ist heute als eine der besten wissenschaftliceh Publikationen Deutschlands gewertet.

Als Lehrer an der Universität Regensburg bemühte sich Göller neue Wege im Kontakt mit seinen Studenten zu finden. Einmal wöchentlich hatte er vor oder nach seiner Vorlesung Gespräch mit seinen Studenten bei einer Tasse Kaffee. Damit wünschte er den Studenten bei ihren Bemühungen für die Lösung ihrer Probleme zu helfen. Einmal monatlich lud er eine Gruppe von seinen Studenten in sein Landhaus in Kirn in Ostbayern um mit ihnen über das Wochenende in der schönen Umgebung der Wiesenlandschaft die Probleme des Universitätsstudiums der Anglistik zu diskutieren. Er führte 35 von seinen Studenten zur Erreichung der Doktorwürde, und 9 zur Habilitation. Er sorgte auch dafür, dass sie nach der Beendigung ihrer Universitätsstudien eine entsprechende Dienststelle bekommen haben. Er blieb auch später mit ihnen in einem fruchtbringenden Kontakt.

Als Universitätslehrer für englische Literaturgeschichte sorgte Göller, dass wichtige englische und amerikanische Texte auch in deutschen Ausgaben erreichbar waren, oft mit seinen Anmerkungen.

Als Forscher arbeitete Göller in allen Zeitabschnitten der englischen Literaturgeschichte, von der altenglischen Periode, über Chaucer und Shakespeare, bis in die Gegenwart, einschliesslich die Zeit nach dem Zweiten Weltkrieg. Er widmete dem späten Mittelalter und der Wiederbelebung der Sage von König Arthur im XX. Jahrhundert grosse Aufmerksamkeit. Göllers Interesse für die Sage von König Arthur wurde in ihm schon von seinem Lehrer Walter Schirmer erweckt: unter seiner Leitung vollendete Göller seine Habilitationsarbeit über König Arthur in der englischen Literautr des späten Mittelalters. Göller publizierte in diesem Gabiet noch die Studien über Die Wappen des Königs Arthurs (1962), The Allterative Morte Arthure: A Reassessment of the Poem (1981), Arthurs Aufstieg zum Heiligen (1989). Göller untersuchte auch die Entwicklung der literarischen Formen in der englischen und amerikanischen Literatur, die Geschichte der altenglischen Elegie sowie die Geschichte der Novelle in der englischen und amerikanischen Literatur. Göllers bedeutendste Leistung in diesem Gebiet ist sein Buch Romance and Novel (19-2), in welchem er die Darstellungsformen in der englischen Literaur von der spätmittelalterlichen Romance und bis zu den Anfängen des modernen Romans untersucht.

Göllers Bibliographie zählt über 100 wissenschaftliche Werke und über 60 Resensionen. ${ }^{3}$ Das ist eine ausserordentlich grosse Zahl wenn man berücksichtigt die Zeit

\footnotetext{
${ }^{3}$ Göllers Bibliographie für die Zeit von 1952 bis 1985 ist publiziert in: Göllers Festsshrift zu seinem 65 Jahresjubiläum (hgg. von Uwe Böker, Manfred Parma und Rainer Schöwerling, Stuttgart 1989, 375-383). - Göllers Publikationen aus der Zeit 1985-2003 sind bibliographisch erfasst in Göller Festschrift zum 80 Geburtsjahr (hgg. von Uwe Böker, Lang Vlg., 2004, Frankfurt a/M, S. 9-16). Göllers Werke aus seinen letzten Lebensjahren sind bibliographisch angeführt im Göllers Nekrolog geschrieben von Richard Utz. S. oben Anm.
} 
in welcher er lebte und die Schwierigheiten, die er umgehen musste, bevor er überhaupt erst die Möglichkeit hatte sich der wissenschaftlichen Arbeit zu widmen. Er war vor allem interessiert in der wissenschaftlich begründeten Deutung des literarischen Textes. Besonders lieb waren ihm Probleme, die anscheinend einfach waren, die jedoch mit der gut begründeten Analyse zur Lösung von Komplexen Fragen führen konnten. Göller erforschte vor allem den Inhalt, weniger die ästhetische Seite eines Werkes, oder seine theoretischen Grundlagen, obgleich er auch mehrmals seine Struktur eingehender besprach.

Ich kann diesen Teil meines Berichtes nur noch mit der Feststellung beschliessen, dass Göller in einer grossen Zahl von verantwortungsvollen Pflichten aktiv war, die ich hier nur kurz aufzählen kann: er organisierte in Regensburg und in Passau mehrere wissenschaftliche Konferenzen deutscher Forscher, an denen auch ausländische Wissenschaftler teilnehmen konnten. Er war Mitglied von mehreren deutschen und internationalen Organisationen, die ihm auch leitende Funktionen anvertraut hatten. Als Gast gab er einzelne Vorträge an verschiedenen Universitäten in Deutschland und im Ausland. Er erhielt Einladungen von den Universitäten Freibung in der Schweiz, Tübingen und Kiel, dass er Lehrstühle für Anglistik an diesen Universitäten übernähme, er akzeptierte jedoch diese Angebote nicht. Er untersuchte Probleme aus der Frühgeschichte Regensburgs und schrieb wissenschaftliche Werke in diesem Gebiet. Er interessierte sich für die Qualität des Unterrichtes der fremden Sprachen in Regensburg und in der Umgebung von Kirn und sorgte für die Nachbildung der Mittelschullehrer. Er war tätig im Schriftstellerverein der Stadt Regensburg.

3.

Zum Schluss müssen wir noch über Göllers Tätigkeit für die Entwicklung guter Beziehungen zwischen den deutschen Universitäten und den Universitäten in anderen Teilen Europas und Amerikas berichten. In Slowenien waren wir in dieser seiner Tätigkeit besonders interessiert.

Als Professor für Anglistik hatte Göller gute Beziehungen mit den Universitäten in Grossbritanien, in den Vereinigten Staaten und in Kanada. An mehreren von diesen Universitäten arbeiteten seine früheren Schüler als Lehrer.

Göller hatte gute Beziehungen mit polnischen Anglisten, besonders mit der Jagiellonischen Universität in Krakau. Als Anerkennung für diese seine Verdienste wurde ihm die Verdienstmedaille der Jagiellonischen Universität verliehen.

Man findet in Göllers Biographien die Angabe, dass Göller auch Kontakte mit jugoslawischen Universitäten pflegte. Das ist nicht ganz richtig: Göller hatte nur engere Kontakte mit Slowenien. Seine Beziehungen mit anderen jugoslawischen Universitäten sind mir unbekannt: wahrscheinlich existierten sie nicht.

In 1977 kam Göller mit einer Gruppe von Universitätsprofessoren aus Regensburg nach Ljubljana um beim Rektorat der hiesigen Universität die Möglichkeit der wissenschaftlichen Beziehungen zwischen den Universitäten Regensburg und Ljubljana zu besprechen. Die Universität Ljubljana fand dieses Angebot interessant, da man von einer neugegründeten deutschen Universität frische Anregungen für solche Kontakte 
erwartete. Man unterzeichnete den Vertrag für die Entwicklung der wissenschaftlichen Zusammenarbeit zwischen den Universitäten Regensburg und Ljubljana. Dieser Vertrag machte es auch mir möglich, dass ich mehrere Plätze in Bayern besuchen konnte, oft mit Göllers Hilfe: Regensburg mit seinen interessanten alten Gabäuden und mit der Walhalla am Ufer der Donau; Passau, Chiemsee und Burghausen. In Altötting war ich überrascht von der Form der dortigen Kirche, die eine grosse Ähnlichkeit mit mehreren Kirchen in Slowenien aufweist; so mit der Kirche des hl. Johannes des Täufers im Orte Muta, im Drautale östlich von Dravograd (Unterdrauburg); und die Kirche an der Strasse Velenje (Wöllan) - Slovenj Gradec (Windischgraz), im Orte Šentilj bei Mislinja (Missling).

\title{
4.
}

Die interessanteste Reise, die ich mit Göller in Slowenien machte, folgte dem Weg den Wolfram von Eschenbach in seinem Ep Parzival dem Trevrizent zuschreibt. Wahrscheinlich ist Wolfram selbst diesen Weg geritten (s. Parziyal, Verse 498, 21-499, 10):

\author{
Ūz Zilje ich vür den Rôhäs reit \\ drî maentage ich dâ vil gestreit \\ mich dûhte ich hat dâ wol gestriten: \\ dar nâch ich schierste kom geriten \\ in die wîten Gandine \\ dâ nâch der ane dîne \\ Gandîn wart genennet. \\ dâ wart Ithêr bekennet. \\ Die selbe stat lît aldâ \\ dâ diu Greian in die Trâ, \\ Mit golde ein wazzer, rinnet, \\ dâ wart Ithêr geminnet. \\ Dîne basen er dâ vant: \\ diu was vrouwe überz lant: \\ Gandîn von Anschouwe \\ hiez si dâ wesen vrouwe. \\ si heizet Lammire: \\ so ist daz lant genennet Stire \\ swer schildes ambet üeben wil, \\ der muoz durchstrîchen lande vil.
}

Von der Mauer der Burgruine Cilli, die sich über den Gipfel des Berges ausbreitet, der sich über die Stadt Celje (Cilli) erhebt, hatten wir eine schöne Aussicht über das weite Tal des Flusses Savinja (Sann). Aus Celje fuhren Göller und ich ostwärts, entlang dem Bach Voglajna, der sich hier seinen Weg, der unserer Reiserichtung entgegengesetzt war, durch enge Täler, umgeben von dichten Wäldern und einzelnen Bauerngütern, fand. 
Wir kamen in den Markt Rogatec (in Wolfram: Rohäs). Die hügelige Umgebung von Rogatec ist beherrscht von einer Anhöhe mit steilen bewaldeten Abhängen und mit einer mittelalterlichen Schlossruine auf der Spitze. Wir stiegen zu der Ruine und fanden an der Seite, die dem Tale zugewendet ist, eine geebnete Fläche mit Wiese bewachsen und durch eine niedrige Mauer von dem steilen bewaldeten Abhange getrennt. Hier waren kleinere ritterliche Kampfspiele möglich. Grössere Turniere konnten nur unten im Tale in der Umgebung des Marktes statt finden.

Von Rogatec fuhren wir weiter über Pässe zwischen den Hügeln und durch enge Täler, umgeben von steilen Wiesen. Endlich kamen wir auf einen Berg mit mehreren Häusern und einer schönen gotischen Kirche. Die Kirche ist mit ihrer beherrschenden Lage schon aus der Ferne sichtbar. Sie stammt aus dem Spätmittelalter und wurde erbaut zum Andenken an die Schlacht bei Nikopol, einer Stadt in Bulgarien auf dem Ufer der Donau. In dieser Schlacht am 26. September 1396 wurde das christiche Heer bestehend aus Kämpfern aus verschiedenen Teilen Europas, darunter vielen Franzosen, vernichtend geschlagen. In dieser Schlacht hat sich Hermann II., Graf von Cilli, mit seiner Tapferkeit und kaltblütiger Geistesgegenwart besonders ausgezeichnet. Die Familie des Grafen gab eine bedeutende Summe Geldes als ihren Beitrag für den Bau dieser Kirche.

Zu Mittag sassen wir nahe der Kirche auf einer von der Sonne bestrahlten Terrasse, die angenehm warm war. Wir bewunderten die schöne Umgebung, von den Weinbergen reich bewachsen. Die Luft war gefüllt mit dem Singen der Grillen und Vögel in welches sich das Klappern der Klopotetz einmischte.

Von dieser Kirche fuhren wir bergab in das Tal des Flusses Drava (Drau) und in die Stadt Ptuj (Pettau). Wolfram nennt in seinem Ep Parzival den Namen dieser Stadt nicht, doch ist sie mit den Namen der Flüsse Greian und Trâ deutlich erkennbar. In der nördlichen Vorstadt der Stadt Ptuj fanden wir den Bach Grajena (in Wolfram Greian), der dann durch die östlichen Vororte von Ptuj in einer tiefen Rinne zu seiner Mündung in den Fluss Drava (deutsch Drau, Wolfram Trâ) fliesst. Wir besuchten noch die mittelalterliche Kirche Hajdina (in Wolfram: Gandin) wo Göller mehrere Photoaufnahmen von dortigen Fresken machte, die er dann in seinem Buche Der Bildtypus Imago Pietatis (Regensburg, o.D. Vgl. Abb. 24 und 25) veröffentlichte.

Von Pettau fuhren wir zurück nach Hause in Ljubljana (Laibach). Unterwegs besuchten wir noch ein Bauerngut in der Umgebung von Cilli (Celje). Die Leute in diesem Haus waren mir noch aus der Vorkriegszeit bekannt. Wir waren freundlich empfangen: man bewirtete uns mit frischgekochten Esskastanien, die im Walde gesammelt waren, und mit Weinmost. Dieser war noch etwas süss, obgleich seine Gärung schon beinahe beendet war. In guter Gesellschaft vergingen uns die Nachmittagsstunden schnell. Als wir spät am Abend nach Laibach zurück kamen, sagte mir Göller, dass er sich schon lange nicht so wohl gefühlt hatte wie in diesem Bauernhaus in der Umgebung von Cilli.

Universität Ljubljana, Slowenien 